\title{
Peer Assisted Study Support (PASS) and Students as Change Agents (SACA) in Mathematics at the University of Nottingham
}

Stephen Cox, School of Mathematical Sciences, University of Nottingham, Nottingham, UK. Email: stephen.cox@nottingham.ac.uk

Laurence Cook, School of Mathematical Sciences, University of Nottingham, Nottingham, UK. Sam Nield, School of Mathematical Sciences, University of Nottingham, Nottingham, UK.

\begin{abstract}
In 2015-16, a Peer Assisted Study Support (PASS) scheme was introduced in Mathematics at the University of Nottingham. A distinctive feature of this PASS scheme is its intimate linking to the University's Nottingham Advantage Award (NAA) scheme, which recognises a wide range of students' extracurricular activities, including serving as a PASS Leader. Furthermore, the PASS scheme has been developed in conjunction with the NAA's Students as Change Agents and Change Leaders (SACA and SACL) programmes, which recognise student-staff partnerships that change teaching and learning practice. Essential to the success of the scheme has been its genesis through such a partnership, in particular two summer internships in 2015 to develop PASS materials, supported by the Sigma Network and the University's Teaching Transformation Programme.
\end{abstract}

Keywords: PASS, transition, student support, student-staff partnership, Nottingham Advantage Award.

\section{Peer Assisted Study Support and Students as Change Agents}

Peer Assisted Study Support-type schemes are nowadays fairly common in university mathematics departments. Kane and Sinka (2009) describe some wide-ranging examples, from specific module support to transition support focussed on providing an introduction to university services. Not only do PASS schemes provide support to first-year students in their transition to university mathematics, they also provide useful curriculum support, and significant CV benefits for the students involved (Hibberd and Grove (2009) discuss the challenges in developing employability skills in maths programmes). In the Nottingham incarnation, PASS groups have regular scheduled meetings consisting of around a dozen first-year students and three higher-year students (the PASS Leaders). The academic staff member responsible (PASS Director) is the first author; between him and the PASS Leaders are five Senior PASS Leaders who help administer the scheme.

The key distinctive feature of the PASS scheme at Nottingham is that it has been developed from the start as a student-staff partnership; this aspect has helped to ensure buy-in from students, and has helped us to tailor the scheme to our students and to fit it to our curriculum. We borrowed the general framework from the successful scheme at the University of Manchester (Walker, 2015) and impetus was provided through the University of Nottingham's Teaching Transformation Programme (TTP). In late 2014/early 2015, the first author collaborated with the student Mathematics Society, MathSoc, then with a focus group of interested students, to begin to shape the scheme. Some of the focus group students were pioneers on the new NAA module Students as Change Agents, which allowed their contributions to scoping and shaping the scheme to be formally recognised by the University. These SACA students went on to be the first generation of Senior PASS Leaders.

\subsection{Organisational principles of PASS}

We would select the PASS groups by combining pairs of first-year tutorial groups (each comprising 5-6 first years). This would have the advantage that right from the start the first years would already 
know some others in their PASS group. Overall, this feature was generally reported by PASS Leaders as contributing positively to the atmosphere of PASS sessions at the start of the academic year. However, one unanticipated slight problem with this arrangement proved to be that in a small number of cases attendance at PASS sessions suffered from a "herd" effect, whereby the absence of one or two key students from one tutorial group would seem to lead to the absence of that entire tutorial group en masse.

PASS groups would meet informally at the start of the new academic year to fulfil a welcoming, mentoring role, equivalent to the Parenting Scheme historically run by MathSoc. This Parenting Scheme had involved higher-year students providing an informal introduction/welcome to the University for the first years. Unfortunately, the lifetime of the "parenting" relationship had typically been very short, often not extending beyond the induction week at the start of the year, and we were keen to improve this.

Formal PASS sessions would not begin until the third week of lectures. This would allow plenty of time for training of PASS Leaders, with some contingency, as this was the first year of operation. With hindsight, this hiatus was a mistake, as students seemed to be eager to start PASS sessions, and concerns regarding the logistics of an earlier start proved unfounded.

PASS sessions would combine some peer mentoring-type discussion with academic work. This decision was in contrast to a University move towards purer Peer Mentoring schemes, and meant that our scheme involved considerably more work to set up. To some extent, this decision to provide an obvious academic purpose to each session was made in the expectation that first-year students would readily appreciate this purpose, and hence engage well with the scheme. It would also improve buy-in from staff, who would recognise the value of the scheme in developing key mathematical skills and knowledge, both in the first-year students and also in the PASS Leaders. The ostensible academic focus of each session was also provided in the knowledge that the interpersonal skills required for effective peer mentoring are not naturally well developed in many of our mathematics students; thus the peer-mentoring aspect could take place within a "safe" mathematical context.

PASS sessions would take place fortnightly, with training sessions in the intervening weeks. This decision was made in part to limit the commitment required of PASS Leaders and in part to limit the number of session plans that needed to be completed for the first year of operation.

Bespoke academic content would be created, free from specific module "branding"; this would allow focus on areas of perceived student weakness (proof, curve sketching, use of definitions, ...) and help to demodularise student thinking, with an eye on trying to address the "Mathematics Problem" (LMS 1995). In fact, somewhat predictably, even though module "branding" was not explicit, anecdotal evidence from PASS Leaders suggested that the first years did tend to assign PASS topics to specific modules, so the attempt to demodularise was only a partial success.

A bespoke Nottingham Advantage Award module would be created for PASS Leaders. This module would be optional and would be assessed through a reflective piece of writing, of 500-1000 words, for which training was provided by the University's Careers and Employability Service. Around onethird of PASS Leaders had cited the existence of this NAA module as a key factor in their decision to apply for the scheme; in the end a much greater proportion - around two-thirds - took the assessment. Even if students do not complete the full Award (which generally requires successful completion of three of its modules), individual NAA modules are recorded with their University transcript. 


\section{Implementation}

Support for the new scheme from students has been overwhelming. Indeed, so many students volunteered to be PASS Leaders that we were able to assign three PASS Leaders to each PASS group, rather than two, as had originally been planned.

Essential to the success of the nascent scheme was the work done over summer 2015 by the present authors in writing PASS materials. Some of these materials were road-tested on other students undertaking summer projects in the School, and then further adapted as a result. The summer interns were supported by generous grants from the sigma Network and the University's TTP. The summer internship proved to be an intensive period of work and provided us with many different challenges each day. We had just six weeks to produce - from a nearly blank page - a comprehensive set of materials to support the PASS sessions which would start in September. We capitalised on this freedom by including topics that the students had found difficult in their own personal experience of first year mathematics, as well as topics recommended by staff and topics that we felt would prove useful in later years but traditionally tended to get rather disregarded by first-year students (noting the findings of LMS (1995)).

One particular synergy emerged among the internship team in devising the seventh PASS session of the year, which had initially been focused simply on the routine calculation of eigenvalues and eigenvectors for matrices. We realised that we were frustrated because we had in addition some unrelated, unused partial sessions written to practise use of the Cayley-Hamilton Theorem and also a bunch of ideas on false proofs left over from the early PASS sessions. Between the three of us, we combined these ideas (wisely or unwisely) into one session. This session would start with a false proof based, quite transparently, around division by zero. Then an apparently unrelated calculation would follow of the eigenvalues and eigenvectors of a matrix $\mathbf{A}$, with one of the eigenvalues turning out to be zero. Finally an attempt would be described at using the Cayley-Hamilton Theorem to calculate the inverse $\mathbf{A}^{-1}$ (which, of course, does not exist). Thus the session would link back to that attempted division by zero at the start, with the moral that division by zero (or multiplication by a nonexistent inverse) may not be quite so easy to spot as one might think.

Timetabling of PASS sessions proved to be (just about) possible, even though it required coordination between timetables of all years and across single and joint honours programmes. We managed to avoid any 9am slots, and kept rooms to a civilised side of our large University Park campus. The only significant gripe expressed by first-year students regarding the scheme has concerned the timing of particular PASS sessions. Dissatisfaction has been expressed both in cases involving a long, contiguous block of contact time for the students and also in cases where the PASS session is isolated from other teaching activities in the timetable (some instances of far-from-optimal scheduling had proved unavoidable, given the complex make-up of the student body participating in the scheme). We have allowed PASS groups to request an alternative time for their sessions, provided they can agree on a better time: in some cases, PASS groups have moved and in others no better time has been found.

PASS Leaders support one another through a dedicated Facebook group, which is used to share insights into the way that sessions might be run and to promote forthcoming events. They receive PASS Leader hoodies, which many proudly wear around campus, enhancing the feeling of community. There have been numerous competitions associated with PASS, including one to generate photos that can be used to promote the scheme internally and at Open Days and Visit Days (see Figure 1). 


\section{Student feedback}

Student feedback has, not surprisingly, been overwhelmingly positive. One important source of feedback came from a poll administered by the Senior PASS Leaders, based on a Google form, which most (137) students completed during their final PASS session before the Christmas vacation. Inter alia, students were asked whether PASS had been useful for them (over $75 \%$ felt so), what the most useful PASS topic had been ("proof", followed closely by "curve sketching") and what they felt about the level of the PASS questions. Intriguingly, over $90 \%$ of the first-year students chose "about right" (rather than "too easy" or "too hard") to describe PASS questions. Our interpretation of the last, rather striking, finding is that, even though some questions may have seemed tough at first sight, the availability of immediate help generated in the minds of the first years the perception that (perhaps with hindsight) the questions had been at the right level.

First-year students greatly value the contact with friendly, more experienced students, especially at the start of the year. They also greatly appreciate the academic support they receive, and recognise the efforts that their PASS Leaders make to help them. PASS seems to generate an enhanced community spirit in the School, perhaps aided by the fact that we are lucky enough to have our own dedicated building.

First-year students say "the best thing about PASS is the opportunity to speak to students who have already been through first year and ask them questions about student life in general as well as maths" and "the mentors can sympathise with the stuff we find hard, where a lecturer might not understand :)". PASS Leaders say "as a result of being a PASS Leader, I would now feel more comfortable and willing to take on leadership roles", "I've really enjoyed the sessions once we get down to the maths and I'm explaining a detailed point to someone. I get a great feeling explaining something to someone or showing somebody that the maths is really cool".

In their NAA reflective assessment, PASS Leaders recognised their role in supporting a diverse student body, saying "we ended up [being] very inclusive, utilising our range of personalities to cater for all", "most of our learning resulted from the ways we adapted to different students' needs", "I have tried to adjust my mentoring style depending on the group", "the biggest challenge was trying to find out the best way to learn for a particular person" and "I have learned how to alter my approach in situations to suit different students and learning styles".

One PASS Leader wrote "from the outset, there was a feeling that everyone involved in PASS is part of a team and as a result I had a real incentive to make the sessions go as well as I possibly could". An important aspect of the scheme is the employability skills that the PASS Leaders pick up - these are difficult to develop elsewhere in a mathematics course; one PASS Leader wrote "these are skills that I have been able to use in job interviews by using PASS as an example [whereas] before this year I have been struggling to find examples of [using] these qualities in real life". 

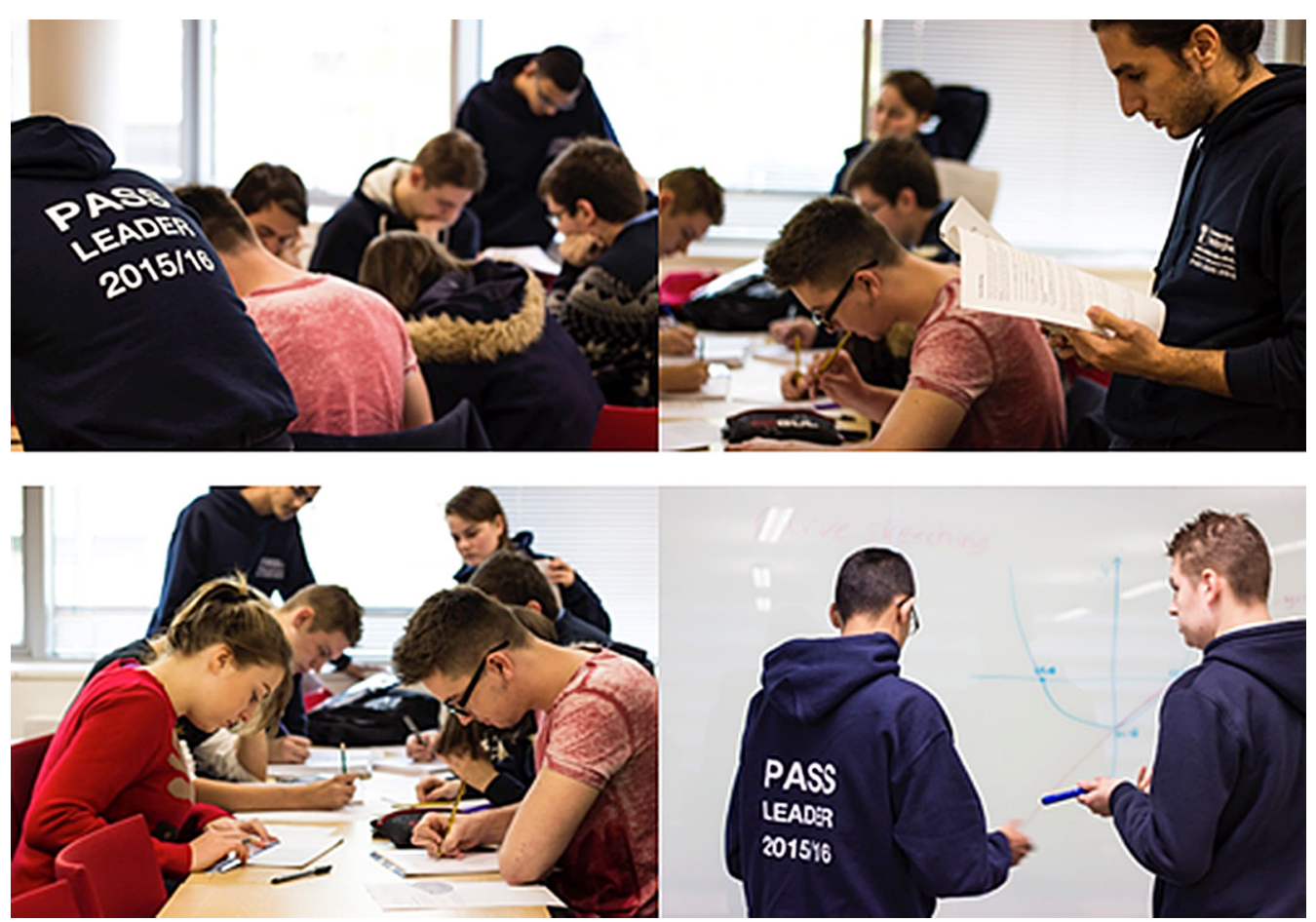

Figure 1. PASS Group R won the photo competition - a PASS session in action!

\section{Future developments}

The PASS scheme provides benefits for students in many ways. First years get support in their transition to university, academically and personally. PASS Leaders and Senior PASS Leaders get significant $\mathrm{CV}$ benefits, whether or not they complete one of the associated NAA modules. PASS also allows students with a passion for mathematics to share that passion in an effective way (Duah, Croft and Inglis, 2013). But there is more to do.

With hindsight, we were too conservative deciding to start the formal PASS sessions so late in the Autumn Term; in future years we would aim to begin them earlier, in the second week of lectures. Student feedback has also consistently asked for more PASS sessions, so we aim to run ten PASS sessions in 2016-17 instead of the eight that ran in 2015-16.

As part of the NAA's Students as Change Leaders programme, this year's Senior PASS Leaders are extending and more properly defining the role of the Senior PASS Leader so that, over time, more responsibility for the running of the scheme can pass from staff to students. Such a transfer of responsibility is valuable for ensuring relevance of the scheme and for providing greater latitude for students to develop the scheme according to their own initiative.

\section{Conclusion}

Success of the PASS scheme at Nottingham owes itself to a number of factors. First, we had strong support both from within our institution and from outside; in particular, there was strong buy-in at all levels in the School of Mathematical Sciences. Also, we developed the scheme from the start as a student-staff partnership, rather than a "top-down" generic imposition. We also provided each session with a clear academic focus. These considerations helped to ensure that the scheme was genuinely meaningful to our students. By working closely with the Nottingham Advantage Award and with the University's Careers and Employability Service, we were able to maximise the careers benefits for those involved. 
We are happy to share our experiences and our materials with colleagues in other institutions. While materials have been designed to fit the timings of the curriculum and other events in the University of Nottingham calendar, they also have quite general features that may be of interest. Interested readers should feel free to contact the first author.

\section{References}

Duah, F., Croft, T. and Inglis, M., 2013. Can peer assisted learning be effective in undergraduate mathematics? Int. J. Math. Education in Science and Technology, 45(4), pp.552-565.

Hibberd, S. and Grove, M., 2009. Developing graduate and employability skills within a mathematical sciences programme. MSOR Connections, 9(2), pp.33-39.

LMS, IMA \& RSS, 1995. Tackling the Mathematics Problem. [pdf] London: LMS. Available at: http://www.mei.org.uk/files/pdf/Tackling_the_Mathematics_Problem.pdf [Accessed 10 April 2016].

Kane, S. and Sinka, I., 2009. Good practice in undergraduate peer support. MSOR Connections, 9(4), pp.11-14.

Walker, L., 2015. Enabling students to become independent learners. In M. Grove, T. Croft, J. Kyle and D. Laswon, eds. Transitions in Undergraduate Mathematics Education. Birmingham: University of Birmingham. 